\title{
On the Integral $\int_{0}^{\pi / 2} \log ^{n} \cos x \log ^{p} \sin x d x$
}

\section{By K. S. Kölbig}

\begin{abstract}
A formula is derived for the integral in the title which allows easy evaluation by formula manipulation on a computer.
\end{abstract}

1. Introduction. In a monograph on generalized polylogarithms, and in a paper on series of reciprocal powers, Nielsen [7], [8] remarked that

$$
r_{n p}=\int_{0}^{\pi / 2} \log ^{n} \cos x \log ^{p} \sin x d x \quad(n \geqslant 0, p \geqslant 0)
$$

can be expressed as $\pi$ times a polynomial in $\eta(q)(1 \leqslant q \leqslant n+p)$, with rational coefficients, where

$$
\eta(q)=\sum_{k=1}^{\infty} \frac{(-1)^{k+1}}{k^{q}}= \begin{cases}\log 2 & (q=1), \\ \left(1-2^{1-q}\right) \zeta(q) & (q>1) .\end{cases}
$$

This polynomial is homogeneous of degree $n+p$ if one considers $\eta(q)$ to be of degree $q$, and if the degree of a product is the sum of the degrees of its factors. $\zeta(q)$ is the Riemann zeta function for integer arguments. Since $r_{n p}=r_{p n}$, it is sufficient to consider $n \geqslant p \geqslant 0$.

In an earlier paper [4], the following complicated closed expression for $r_{n p}$ was derived:

$$
r_{n p}=\frac{\pi n ! p !}{2^{n+p+1}} \sum_{k=1}^{n+p} \frac{1}{k !} \sum_{\left\{p_{i}\right\}} \sum_{\left\{n_{i}\right\}} f\left(p_{1}, n_{1}\right) \cdots f\left(p_{k}, n_{k}\right),
$$

where the innermost sums run over all sets $\left\{p_{i}\right\},\left\{n_{i}\right\}$, which satisfy the conditions

$$
p_{i} \geqslant 0, \quad \sum_{i=1}^{k} p_{i}=p ; \quad n_{i} \geqslant 0, \quad \sum_{i=1}^{k} n_{i}=n,
$$

and where the function $f(r, s)$ is given by

$$
\begin{aligned}
f(r, s)= & \left(1-\delta_{0 r}\right)\left(1-\delta_{0 s}\right) \frac{(-1)^{r+s+1}}{r+s}\left(\begin{array}{c}
r+s \\
r
\end{array}\right) \zeta(r+s) \\
& +\left|\delta_{0 r}-\delta_{0 s}\right| \xi(r+s)
\end{aligned}
$$

where

$$
\xi(q)= \begin{cases}-2 \log 2, & q=1 \\ (-1)^{q} \frac{2^{q}-2}{2} \zeta(q), & q>1\end{cases}
$$

and $\delta_{i j}$ is the Kronecker symbol.

Received June 21, 1982.

1980 Mathematics Subject Classification. Primary 33A15, 33A10.

(C)1983 American Mathematical Society

0025-5718/82/0000-0899/\$02.00 
The actual computation of $r_{n p}$ from (3), even for small $n$ and $p$, is quite complicated. Explicit expressions for $r_{n p}(1 \leqslant n \leqslant 4,0 \leqslant p \leqslant n)$ in terms of $\eta(q)$, computed from (3), have been given in [4]. It may be noted that, in the relevant handbooks, only expressions for $r_{n 0}$ or $r_{0 n}$ with $n=1,2$ are listed.*

2. An Expression for $r_{n p}$. It is the purpose of this note to derive another expression for $r_{n p}$ which is well-adapted to evaluation by a formula-manipulation system such as REDUCE [2]. As in the derivation of (3), we start from the definition (1) and, after substituting $t=\cos ^{2} x$ in (1), express $r_{n p}$ as a derivative of Euler's beta function:

$$
\begin{aligned}
r_{n p} & =\left.\frac{1}{2^{n+p+1}} \frac{\partial^{n+p}}{\partial \beta^{n} \partial \alpha^{p}} \int_{0}^{1} t^{\beta-1 / 2}(1-t)^{\alpha-1 / 2} d t\right|_{\alpha=\beta=0} \\
& =\left.\frac{1}{2^{n+p+1}} \frac{\partial^{n+p}}{\partial \beta^{n} \partial \alpha^{p}} \frac{\Gamma\left(\frac{1}{2}+\alpha\right) \Gamma\left(\frac{1}{2}+\beta\right)}{\Gamma(1+\alpha+\beta)}\right|_{\alpha=\beta=0}
\end{aligned}
$$

We introduce the power series [6]

$$
\begin{aligned}
& \Gamma(1+x)=\sum_{k=0}^{\infty} b_{k} x^{k} \quad(|x|<1), \\
& 1 / \Gamma(1+x)=\sum_{k=0}^{\infty} a_{k} x^{k} \quad(|x|<\infty),
\end{aligned}
$$

where $a_{0}=b_{0}=1$,

$$
\begin{aligned}
& a_{k}=-\frac{1}{k} \sum_{m=1}^{k}(-1)^{m} \tilde{\zeta}(m) a_{k-m}, \\
& b_{k}=\frac{1}{k} \sum_{m=1}^{k}(-1)^{m} \tilde{\zeta}(m) b_{k-m} \quad(k>0),
\end{aligned}
$$

and $\tilde{\zeta}(1)=\gamma$ (Euler's constant), $\tilde{\zeta}(m)=\zeta(m)$ for $m \geqslant 2$. A direct approach by setting $x=-\frac{1}{2}+\alpha$ and $x=-\frac{1}{2}+\beta$ in (8) and then carrying out the differentiations in (7) does not lead to a satisfactory result; the resulting expression would contain infinite series, and the expected quantities $\pi$ and $\log 2$ would appear only implicitly. We therefore take a different route and apply the duplication formula [5]

$$
\Gamma(2 x)=\frac{1}{\sqrt{2 \pi}} 2^{2 x-1 / 2} \Gamma(x) \Gamma\left(\frac{1}{2}+x\right),
$$

so that from (7)

$$
r_{n p}=\left.\frac{\pi}{2^{n+p+1}} \frac{\partial^{n+p}}{\partial \beta^{n} \partial \alpha^{p}} \frac{2^{-2 \alpha-2 \beta}}{\Gamma(1+\alpha+\beta)} \frac{\Gamma(1+2 \alpha)}{\Gamma(1+\alpha)} \frac{\Gamma(1+2 \beta)}{\Gamma(1+\beta)}\right|_{\alpha=\beta=0}
$$

We have separated out the factor $\pi$, and the terms involving $\log 2$ will arise from the differentiation of $2^{-2 \alpha-2 \beta}$.

The differentiations with respect to $\alpha$ and $\beta$ may now be carried out in (11). The result is a complicated six-fold sum in a form suitable for evaluation by formula

*Nielsen [8] made a similar comment. 
manipulation. A much simpler expression can, however, be obtained as follows. We rewrite (11) as

$$
r_{n p}=\left.\frac{\pi}{2^{n+p+1}} \frac{\partial^{n+p}}{\partial \beta^{n} \partial \alpha^{p}} \frac{G(\alpha) G(\beta)}{\Gamma(1+\alpha+\beta)}\right|_{\alpha=\beta=0},
$$

where

$$
G(x)=2^{-2 x} \frac{\Gamma(1+2 x)}{\Gamma(1+x)},
$$

and develop $G(x)$ as a power series. Using the series [6]

$$
\log \Gamma(1+x)=-\gamma x+\sum_{k=2}^{\infty}(-1)^{k} \zeta(k) x^{k} / k \quad(|x|<1)
$$

we find

$$
\text { (15) } \log G(x)=-(\gamma+2 \log 2) x+\sum_{k=2}^{\infty}(-1)^{k} \zeta(k) \frac{2^{k}-1}{k} x^{k} \quad\left(|x|<\frac{1}{2}\right) \text {. }
$$

In order to obtain a series for $G(x)$ from (15), we make use of the theorem that if

$$
f(x)=\sum_{k=1}^{\infty} a_{k} x^{k}
$$

is a formal power series with $a_{0}=0$, then

$$
e^{f(x)}=\sum_{k=0}^{\infty} c_{k} x^{k}
$$

where the coefficients $c_{k}$ are given recursively by

$$
c_{k}=\frac{1}{k} \sum_{m=1}^{k} m a_{m} c_{k-m} \quad(k>0) .
$$

This theorem can be proved analogously to Theorem $1.6 \mathrm{c}$ of Henrici [3,p. 42]. Applying this result to the series (15), we find

$$
G(x)=\sum_{k=0}^{\infty} b_{k}^{*} x^{k} \quad\left(|x|<\frac{1}{2}\right),
$$

where $b_{0}^{*}=1$,

$$
b_{k}^{*}=\frac{1}{k} \sum_{m=1}^{k}(-1)^{m} \zeta^{*}(m) b_{k-m}^{*}
$$

and

$$
\zeta^{*}(m)= \begin{cases}\gamma+2 \log 2, & m=1 \\ \left(2^{m}-1\right) \zeta(m), & m>1\end{cases}
$$

We now differentiate (12) with respect to $\alpha$ and obtain

$$
\begin{aligned}
H(\beta) & =\left.\frac{\partial^{p}}{\partial \alpha^{p}} \frac{G(\alpha)}{\Gamma(1+\alpha+\beta)}\right|_{\alpha=0}=\left.\sum_{\rho=0}^{p}\left(\begin{array}{l}
p \\
\rho
\end{array}\right) \Gamma^{-1}(1+\alpha+\beta)^{(\rho)} G(\alpha)^{(p-\rho)}\right|_{\alpha=0} \\
& =p ! \sum_{\rho=0}^{p} b_{p-\rho}^{*} \sum_{k=\rho}^{\infty} a_{k}\left(\begin{array}{l}
k \\
\rho
\end{array}\right) \beta^{k-\rho}
\end{aligned}
$$


Similarly,

$$
\left.\frac{\partial^{n}}{\partial \beta^{n}} H(\beta) G(\beta)\right|_{\beta=0}=\sum_{\nu=0}^{n}\left(\begin{array}{c}
n \\
\nu
\end{array}\right) H(\beta)^{(\nu)} G(\beta)^{(n-\nu)}
$$

and therefore finally

$$
r_{n p}=\frac{\pi n ! p !}{2^{n+p+1}} \sum_{\nu=0}^{n} b_{n-\nu}^{*} \sum_{\rho=0}^{p}\left(\begin{array}{c}
\nu+\rho \\
\rho
\end{array}\right) b_{p-\rho}^{*} a_{\nu+\rho} .
$$

This expression, although still complicated and revealing less of the structure of $r_{n p}$ than formula (3), is much more suitable for actual computation. Using a formula manipulation system, the evaluation of (22) is in fact straightforward once the expressions for $a_{k}(0<k<n+p)$ and $b_{k}^{*}(0<k<\max (n, p))$ have been initially established. It follows from (5) that, at least, all terms involving $\tilde{\zeta}(1)=\gamma$ will cancel in the final expression for (22). For the case $n \geqslant 0, p=0,(22)$ reduces to

$$
r_{n 0}=\pi \frac{n !}{2^{n+1}} \sum_{\nu=0}^{n} b_{n-\nu}^{*} a_{\nu}
$$

which is another form of Bowman's determinant [1] for $r_{n 0}$. The result of Bowman for $r_{n 0}$ can also be found in the book by Lewin [5].

3. A Table for $r_{n p}$. We give here, as examples, the expressions for $r_{n p}$ for $n=1,2$ and $0 \leqslant p \leqslant n$. In order to complete the table of $r_{n p}$ given in [4], we also present expressions for ${ }_{n p}$ for $n=5$ and $0 \leqslant p \leqslant 5$ in Table 1. Note that Nielsen has already given the expressions for $r_{20}, r_{11}, r_{30}$ in [9], for $r_{02}, r_{11}, r_{03}$ in [10], and for $r_{12}, r_{22}, r_{13}$ in [11], derived by different methods.

$$
\begin{aligned}
r_{10} & =-\frac{1}{2} \pi \log 2, \\
r_{11} & =\frac{\pi}{8}\left(-\zeta(2)+4 \log ^{2} 2\right)=\frac{\pi}{2}\left(-\frac{1}{24} \pi^{2}+\log ^{2} 2\right), \\
r_{20} & =\frac{\pi}{4}\left(\zeta(2)+2 \log ^{2} 2\right)=\frac{\pi}{2}\left(\frac{1}{12} \pi^{2}+\log ^{2} 2\right) \\
r_{21} & =\frac{\pi}{8}\left(\zeta(3)-4 \log ^{3} 2\right)=\frac{\pi}{2}\left(-\log ^{3} 2+\frac{1}{4} \zeta(3)\right) \\
r_{22} & =\frac{\pi}{16}\left(-3 \zeta(4)-8 \zeta(3) \log 2+3 \zeta^{2}(2)+8 \log ^{4} 2\right) \\
& =\frac{\pi}{2}\left(\frac{1}{160} \pi^{4}+\log ^{4} 2-\zeta(3) \log 2\right)
\end{aligned}
$$

Numerical values of $r_{n p}$ for $0 \leqslant n \leqslant 5,0 \leqslant n \leqslant p$, with 21 digits are given in Table 2 . 


\section{TABLE 1}

$$
\begin{aligned}
& r_{50}=-\frac{\pi}{8}\left(90 \zeta(5)+105 \zeta(4) \log 2+30 \zeta(2) \zeta(3)+60 \zeta(3) \log ^{2} 2\right. \\
& \left.+15 \zeta^{2}(2) \log 2+20 \zeta(2) \log ^{3} 2+4 \log ^{5} 2\right) \\
& r_{51}=\frac{\pi}{32}\left(-30 \zeta(6)+300 \zeta(5) \log 2-135 \zeta(2) \zeta(4)-60 \zeta^{2}(3)+360 \zeta(4) \log ^{2} 2\right. \\
& \left.-60 \zeta(2) \zeta(3) \log 2-15 \zeta^{3}(2)+200 \zeta(3) \log ^{3} 2+60 \zeta(2) \log ^{4} 2+16 \log ^{6} 2\right) \\
& r_{52}=\frac{\pi}{32}(90 \zeta(7)+210 \zeta(6) \log 2-150 \zeta(2) \zeta(5)+165 \zeta(3) \zeta(4) \\
& -120 \zeta(5) \log ^{2} 2+90 \zeta(2) \zeta(4) \log 2+210 \zeta^{2}(3) \log 2-105 \zeta^{2}(2) \zeta(3) \\
& -240 \zeta(4) \log ^{3} 2+120 \zeta(2) \zeta(3) \log ^{2} 2-30 \zeta^{3}(2) \log 2 \\
& \left.-140 \zeta(3) \log ^{4} 2-48 \zeta(2) \log ^{5} 2-16 \log ^{7} 2\right) \\
& r_{53}=\frac{\pi}{64}\left(-630 \zeta(8)-1440 \zeta(7) \log 2-165 \zeta(2) \zeta(6)+990 \zeta(3) \zeta(5)-180 \zeta^{2}(4)\right. \\
& -1680 \zeta(6) \log ^{2} 2+720 \zeta(2) \zeta(5) \log 2+360 \zeta(3) \zeta(4) \log 2 \\
& -360 \zeta^{2}(2) \zeta(4)+405 \zeta(2) \zeta^{2}(3)-600 \zeta(5) \log ^{3} 2+180 \zeta(2) \zeta(4) \log ^{2} 2 \\
& +360 \zeta^{2}(2) \zeta(3) \log 2-60 \zeta^{4}(2)+60 \zeta(4) \log ^{4} 2+120 \zeta(2) \zeta(3) \log ^{3} 2 \\
& \left.+60 \zeta^{3}(2) \log ^{2} 2+168 \zeta(3) \log ^{5} 2+60 \zeta^{2}(2) \log ^{4} 2+88 \zeta(2) \log ^{6} 2+32 \log ^{8} 2\right) \\
& r_{54}=\frac{\pi}{64}(2520 \zeta(9)+5670 \zeta(8) \log 2+540 \zeta(2) \zeta(7)+210 \zeta(3) \zeta(6) \\
& -4230 \zeta(4) \zeta(5)+6480 \zeta(7) \log ^{2} 2+1260 \zeta(2) \zeta(6) \log 2 \\
& -3960 \zeta(3) \zeta(5) \log 2-4455 \zeta^{2}(4) \log 2-450 \zeta^{2}(2) \zeta(5)+990 \zeta(2) \zeta(3) \zeta(4) \\
& +630 \zeta^{3}(3)+5040 \zeta(6) \log ^{3} 2-720 \zeta(2) \zeta(5) \log ^{2} 2 \\
& -6120 \zeta(3) \zeta(4) \log ^{2} 2+270 \zeta^{2}(2) \zeta(4) \log 2+1260 \zeta(2) \zeta^{2}(3) \log 2 \\
& -210 \zeta^{3}(2) \zeta(3)+2280 \zeta(5) \log ^{4} 2-1440 \zeta(2) \zeta(4) \log ^{3} 2 \\
& -1680 \zeta^{2}(3) \log ^{3} 2+360 \zeta^{2}(2) \zeta(3) \log ^{2} 2-45 \zeta^{4}(2) \log 2 \\
& +432 \zeta(4) \log ^{5} 2-840 \zeta(2) \zeta(3) \log ^{4} 2-112 \zeta(3) \log ^{6} 2 \\
& \left.-144 \zeta^{2}(2) \log ^{5} 2-96 \zeta(2) \log ^{7} 2-32 \log ^{9} 2\right) \\
& r_{55}=\frac{\pi}{256}(-45360 \zeta(10)-100800 \zeta(9) \log 2-9450 \zeta(2) \zeta(8)-3600 \zeta(3) \zeta(7) \\
& +5400 \zeta(4) \zeta(6)+73800 \zeta^{2}(5)-113400 \zeta(8) \log ^{2} 2-21600 \zeta(2) \zeta(7) \log 2 \\
& -8400 \zeta(3) \zeta(6) \log 2+169200 \zeta(4) \zeta(5) \log 2-1800 \zeta^{2}(2) \zeta(6) \\
& +39600 \zeta(2) \zeta(3) \zeta(5)-33075 \zeta(2) \zeta^{2}(4)-33300 \zeta^{2}(3) \zeta(4) \\
& -86400 \zeta(7) \log ^{3} 2-25200 \zeta(2) \zeta(6) \log ^{2} 2+79200 \zeta(3) \zeta(5) \log ^{2} 2 \\
& +89100 \zeta^{2}(4) \log ^{2} 2+18000 \zeta^{2}(2) \zeta(5) \log 2-39600 \zeta(2) \zeta(3) \zeta(4) \log 2 \\
& -25200 \zeta^{3}(3) \log 2-6750 \zeta^{3}(2) \zeta(4)+15300 \zeta^{2}(2) \zeta^{2}(3) \\
& -50400 \zeta(6) \log ^{4} 2+9600 \zeta(2) \zeta(5) \log ^{3} 2+81600 \zeta(3) \zeta(4) \log ^{3} 2 \\
& -5400 \zeta^{2}(2) \zeta(4) \log ^{2} 2-25200 \zeta(2) \zeta^{2}(3) \log ^{2} 2+8400 \zeta^{3}(2) \zeta(3) \log 2 \\
& -765 \zeta^{5}(2)-18240 \zeta(5) \log ^{5} 2+14400 \zeta(2) \zeta(4) \log ^{4} 2 \\
& +16800 \zeta^{2}(3) \log ^{4} 2-4800 \zeta^{2}(2) \zeta(3) \log ^{3} 2+900 \zeta^{4}(2) \log ^{2} 2 \\
& -2880 \zeta(4) \log ^{6} 2+6720 \zeta(2) \zeta(3) \log ^{5} 2+640 \zeta(3) \log ^{7} 2 \\
& \left.+960 \zeta^{2}(2) \log ^{6} 2+480 \zeta(2) \log ^{8} 2+128 \log ^{10} 2\right)
\end{aligned}
$$




\section{TABLE 2}

$\begin{array}{ll}n & p \\ 0 & 0 \\ 1 & 0 \\ 1 & 1 \\ 2 & 0 \\ 2 & 1 \\ 2 & 2 \\ 3 & 0 \\ 3 & 1 \\ 3 & 2 \\ 3 & 3 \\ 4 & 0 \\ 4 & 1 \\ 4 & 2 \\ 4 & 3 \\ 4 & 4 \\ 5 & 0 \\ 5 & 1 \\ 5 & 2 \\ 5 & 3 \\ 5 & 4 \\ 5 & 5\end{array}$

\begin{tabular}{rrrrr}
\multicolumn{5}{c}{$r_{n p}$} \\
1.57079 & 63267 & 94896 & 61923 & $\mathrm{E}+00$ \\
-1.08879 & 30451 & 51801 & 06525 & $\mathrm{E}+00$ \\
1.08729 & 73195 & 40018 & 85202 & $\mathrm{E}-01$ \\
2.04662 & 20244 & 72740 & 64617 & $\mathrm{E}+00$ \\
-5.10672 & 58055 & 97432 & 35017 & $\mathrm{E}-02$ \\
1.01152 & 43866 & 77157 & 15298 & $\mathrm{E}-02$ \\
-6.04188 & 29097 & 75093 & 52215 & $\mathrm{E}+00$ \\
4.38609 & 79674 & 10765 & 45916 & $\mathrm{E}-02$ \\
-4.26758 & 40717 & 27544 & 48003 & $\mathrm{E}-03$ \\
1.02686 & 60292 & 42556 & 73947 & $\mathrm{E}-03$ \\
2.40528 & 86060 & 94622 & 94399 & $\mathrm{E}+01$ \\
-5.42161 & 86358 & 95971 & 92894 & $\mathrm{E}-02$ \\
2.78905 & 89366 & 54715 & 61317 & $\mathrm{E}-03$ \\
-4.11669 & 99312 & 01587 & 89077 & $\mathrm{E}-04$ \\
1.08779 & 55160 & 65204 & 48571 & $\mathrm{E}-04$ \\
-1.20085 & 59155 & 37857 & 31652 & $\mathrm{E}+02$ \\
8.67856 & 96358 & 57061 & 30428 & $\mathrm{E}-02$ \\
-2.46254 & 98260 & 35781 & 37595 & $\mathrm{E}-03$ \\
2.32819 & 02210 & 25192 & 62212 & $\mathrm{E}-04$ \\
-4.23231 & 23761 & 73142 & 19272 & $\mathrm{E}-05$ \\
1.18188 & 78861 & 42353 & 10303 & $\mathrm{E}-05$
\end{tabular}

European Organization for Nuclear Research

CERN

CH-1211 Geneva 23, Switzerland

1. F. Bowman, "Note on the integral $\int_{0}^{\pi / 2}(\log \sin \theta)^{n} d \theta$," J. London Math. Soc., v. 22, 1947, pp. $172-173$

2. A. C. Hearn, REDUCE Users Manual, 2nd ed., Univ. of Utah, Salt Lake City, Utah, 1973.

3. P. Henrici, Applied and Computational Complex Analysis, Vol. 1, Wiley, New York, 1974.

4. K. S. KOLBIG, "On the value of a logarithmic-trigonometric integral," $B I T$, v. 11, 1972, pp. 21-28.

5. L. LewIN, Polylogarithms and Associated Functions, North-Holland, New York, 1981.

6. Y. L. LuKE, Mathematical Functions and Their Evaluation, Academic Press, New York, 1975.

7. N. NIELSEN, "Der Eulersche Dilogarithmus und seine Verallgemeinerungen," Nova Acta Leopoldina, v. 90,1909, pp. 123-211.

8. N. Nielsen, "Undersøgelser over Reciproke Potenssummer og dere Anvendelse paa Raekker og Integraler," Danske Vid. Selsk. Skr., 6. Raekke, naturvid. og math. Afd., v. 8, 1898, pp. 395-443.

9. N. Nielsen, "Sur la transformation d'une intégrale définie," Oversigt Danske Vid. Selsk. Forh., 1896, pp. 335-347.

10. N. NielSen, "Sur la sommation de quelques séries," Oversigt Danske Vid. Selsk. Forh., 1896, pp. $348-361$.

11. N. Nielsen, “Théorème sur les intégrales $\int_{0}^{\pi / 4} \log ^{p} \sin 2 \phi d \phi$ et $\int_{0}^{\pi / 4} \operatorname{tg} \phi \log ^{P} \sin 2 \phi d \phi$," Oversigt Danske Vid. Selsk. Forh., 1897, pp. 197-206. 